Research Article

\title{
Conditional Symmetry of a Memristive System with Amplitude and Frequency Modulation Control
}

\author{
Hongyan Zang $\mathbb{D}^{D}$, Lili Huang, Tengfei Lei $\mathbb{D}^{\circ}$, and Yanling Wang \\ Collaborative Innovation Center of Memristive Computing Application (CICMCA), Qilu Institute of Technology, \\ Jinan 250200, China \\ Correspondence should be addressed to Tengfei Lei; leitengfeicanhe@126.com
}

Received 15 April 2021; Accepted 5 July 2021; Published 17 July 2021

Academic Editor: Zenghui Wang

Copyright $(92021$ Hongyan Zang et al. This is an open access article distributed under the Creative Commons Attribution License, which permits unrestricted use, distribution, and reproduction in any medium, provided the original work is properly cited.

In this study, we studied the effects of offset boosting on the memristive chaotic system. A system with symmetry and conditional symmetry was constructed, by adding the absolute value function to an offset boosting system. It is proved that the symmetric system or a conditionally symmetric system can be constructed with similar or the same dynamic characteristics by using certain correction and offset boosting in an asymmetric system. In addition to multiple stability, the memristive system can also realize the amplitude and frequency control by introducing a parameter. The simulation circuit verifies the amplitude modulation characteristics of the system.

\section{Introduction}

As the fourth basic circuit element, the memristor has attracted an extensive attention in the nonlinear field. In 1971, Chua proposed a memristor to represent the relationship between charge and flux from the view of symmetry when analyzing the relation among the voltage, current, charge, and flux. In 2008, HP laboratory released a $\mathrm{TiO}_{2}$ charged memristor [1], declaring that a memristor was found. A large number of research studies on memristor models and typical chaotic systems have been published, making the memristor widely studied in secure communication $[2,3]$, cryptography $[4,5]$, image encryption $[6,7]$, associative memory $[8,9]$, voice encryption $[10]$, and recognition and sequencing application [11]. Wang et al. [12] reported a special bursting behavior previously unobserved in third-order autonomous memristive circuits. Wang et al. [13] developed a simple scheme implementing a multisegment piecewise-linear Chua's diode and an inductor-free multiscroll Chua's circuit. In addition, the application of the memristor in artificial synapses has also been started, which is expected to be used for memristor calculation or system design. Many characteristics have also been widely studied, such as hidden attractors [14-17], coexisting attractors [18], multistability [19-21], symmetry [22, 23], amplitude control $[24,25]$, and offset boosting [26-29]. In [30], a simple parametric control scheme for generation of the multiscroll chaotic attractor from the jerk system has been presented. In [31], a modified Chua's circuit with a 5-segment piecewiselinear Chua's diode is presented, in which hidden attractors and coexisting attractors are identified.

Many scientists have shown that some signals do not require additional peripherals to provide pretuning, such as amplitude controllable, frequency controllable [32], and conditional symmetric attractors $[23,33,34]$ in an asymmetric system, extremely multistability attractors [35-37], and so on.

In [38], Li and Sprott researched on amplitude of a chaos dynamical system. The chaos dynamical system can be controlled by the coefficient of one or more terms without changing the dynamics. In [39], cosine function was introduced to keep the polarity balance and conditional symmetric coexisting chaotic attractors. It was found that the initial condition can be used to change the starting oscillation as well as the amplitude and frequency [40]. The output polarity of the signal can be adjusted by adding a DC voltage source [41]. As is known to all, asymmetric systems give coexisting attractors from different directions. Offset 
boosting can be used to construct conditional symmetry to achieve polar balance.

In this study, an offset boosting chaotic system was constructed with the following properties: being of conditional symmetry, amplitude modulation, and frequency modulation. In Section 2, the model of the memristive chaotic system is introduced and its dynamical properties are investigated. In Section 4, amplitude modulation and frequency modulation are described. In Section 5, bifurcation and coexisting attractors are analyzed. In Section 6, circuit implementation is discussed. Section 7 gives conclusion and discussion.

\section{System Model Description}

2.1. Mathematical Model. A new chaotic system is obtained based on the chaotic system variable boosting VB24 [42]. The chaotic system (VB24) has only one linear term, one constant term, and four quadratic terms. We added an absolute value function to VB24. Its new system mathematical model is expressed as

$$
\left\{\begin{array}{l}
\dot{x}=a z^{2}-y z \\
\dot{y}=z^{2}-b \\
\dot{z}=x-c z|z|
\end{array}\right.
$$

where $x, y$, and $z$ are the three dynamical variables, and $a$ and $c$ are all positive parameters. $b$ is a nonzero constant term. In system (1), an absolute value memristor is introduced to construct a four-dimensional memristor chaotic system (2).

$$
\left\{\begin{array}{l}
\dot{x}=a z^{2}-y z \\
\dot{y}=z^{2}-b+W(w)|z| \\
\dot{z}=x-c z|z| \\
\dot{w}=0.5 z-w .
\end{array}\right.
$$

The variable constraint relation of memristor is as follows:

$$
\left\{\begin{array}{cl}
W(w) & =-0.2+|w| \\
\dot{w} & =0.5 z-w .
\end{array}\right.
$$

The parameters of the system (3) are $a=1.5, b=0.7$, $c=0.5$, and initial condition (IC) is $\left(\begin{array}{llll}1 & 0 & 0 & 0\end{array}\right)$. The Lyapunov exponents are $\mathrm{LE} 1=0.117031, \quad \mathrm{LE} 2=0.000306$, $\mathrm{LE}=-0.401139$, and LE4 $=-1.190722$, when time step is $1 \mathrm{~s}$ and time length is $500 \mathrm{~s}$. Numerical simulation software MATLAB is used to numerical simulation, and the phase trajectories of each plane are shown in Figure 1. The attractor of system (1) is asymmetric attractor; it has no symmetry about any coordinate axis and does not have reflection symmetry, rotation symmetry, and inversion symmetry.

2.2. Dynamical Analysis. With conventional dynamical analysis tools, such as bifurcation diagram and Lyapunov exponent spectrum, the dynamical behavior of the chaotic system are explored under the varying parameters $a, b$, and $c$.
Under the conditions of the fixed parameters $b=0.7$, $c=0.5$, and the fixed initial value, Lyapunov exponent and the corresponding bifurcation diagram of the state variable $w$ are shown in Figure 2, when the parameter $a$ increases gradually from 0 to 5 . Both the unstable and stable regions obtained by the Lyapunov exponent spectrum are consistent with those obtained by the bifurcation diagram in Figure 2. In the interval $a \in[0,2.9]$, the system is mainly in a chaotic state and there is a small window of the periodic orbit. The attractor gradually transitions from double to a single scroll attractor. For example, when $a=0.3$, the system is double scroll, $a=1.5$, the system is single scroll, and $a=0.9$, the negative scroll become weaker. When $a \in[2.95,3.8] \cup[4.2,4.3] \cup[4.8,5]$, the system is a limit cycle. For example, when $a=3.5$, the system is periodic of cycle $1, a=4.5$, the system is periodic of cycle 2 , and $a=4.4$, the attractor become sink. For different values of $a$, several typical phase diagrams of the $x-w$ plane with respect to parameter $a$ are shown in Figure 3.

When $c$ varies from 0 to 3 and other parameters are unchanged under the initial condition $(1,0,0,0)$, the bifurcation diagram of the state variable $w$ and the corresponding Lyapunov exponent spectrum are shown in Figure 4, respectively. As shown in Figure 4(a), the system is chaotic when $c \in[0,1.1] \cup[2.85,3]$, and the largest Lyapunov exponent is positive. When $c \in[1.4,1.5] \cup[1.7,1.8] \cup[2,2.8]$, system (2) is periodic. When $c \in[1.2,1.35] \cup[1.8,2]$, the system drops into an interval of chaotic and periodic states alternate with each other. For different values of $c$, several typical phase diagrams of the $x-w$ plane with respect to parameter $a$ are shown in Figure 5.

\section{Symmetry and Conditional Symmetry}

3.1. Symmetry. By using $z|z|$ to replace $z^{2}$ in $x$-dimension, a new four-dimensional memristive chaotic system is designed, and its state equation is

$$
\left\{\begin{array}{l}
\dot{x}=a z|z|-y z, \\
\dot{y}=z^{2}-b+(-0.2+|\varphi|)|z|, \\
\dot{z}=x-c z|z|, \\
\dot{w}=0.5 z-w .
\end{array}\right.
$$

After the transformation of $(x, y, z, w) \longrightarrow(-x, y,-z,-w)$, system (4) has a three-dimensional inversion symmetry property relative to $x-w-z$. The two attractor phase orbital diagrams with inversion symmetry are shown in Figure 6.

3.2. Conditional Symmetry. Here, the absolute value functions $F(x)$ and $F(y)$ are introduced to replace the variables $x$ and $y$. The new system can be described as

$$
\left\{\begin{array}{l}
\dot{x}=a z^{2}-F(y) z, \\
\dot{y}=z^{2}-b+(-0.2+|w|)|z|, \\
\dot{z}=F(x)-c z|z|, \\
\dot{w}=0.5 z-w .
\end{array}\right.
$$




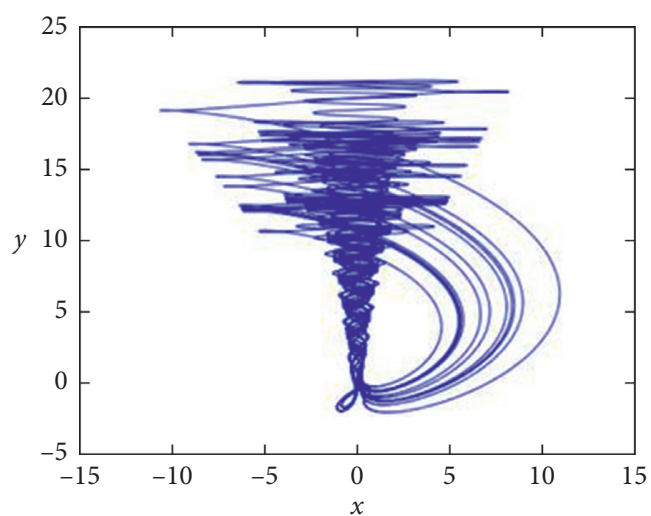

(a)

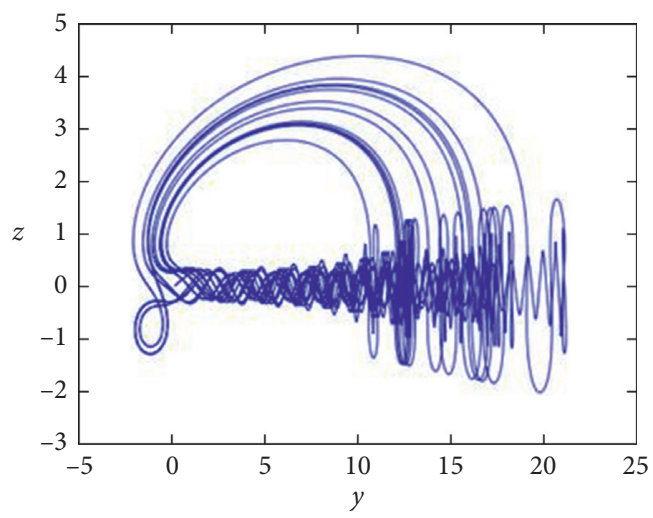

(c)

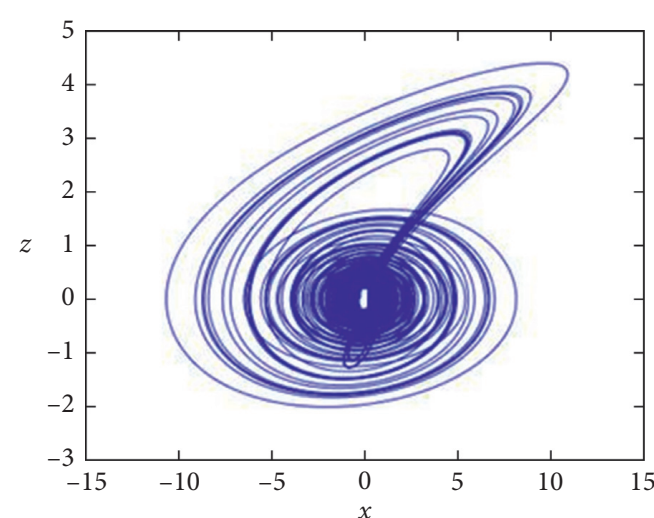

(b)

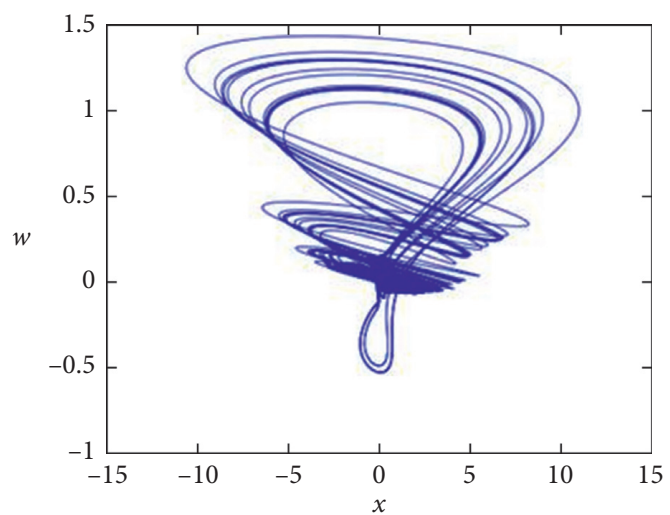

(d)

Figure 1: Phase trajectory diagram of system (2).

The new absolute value function $F(x)=|x|-d$ meet $F(x+d)=-F(x) \quad$ and $\quad F(y)=|y|-e \quad$ meet $F(y+e)=-F(y)$. The increase of two absolute value functions not only does not change the dynamic characteristics of the original system but also brings additional conditional symmetry properties. Figure 7 shows two chaotic attractor phase diagrams with conditional symmetry under the transformation of $x \longrightarrow x+d, y \longrightarrow y+e$, $z \longrightarrow-z$, and $w \longrightarrow-w$, when $a=1.5, b=0.7, c=0.5$, $d=35$, and $e=50$. The original asymmetric system now has symmetry, but it is different from other systems where two attractors are in two systems. System (5) only needs to change the initial state of the system to achieve the coexistence symmetry.

\section{Amplitude Modulation and Frequency Modulation}

The system (5) is scaled by $x \longrightarrow x / k, y \longrightarrow y / k, z \longrightarrow z / k$, $w \longrightarrow w, t \longrightarrow k t$, and $b \longrightarrow k^{2} b$, and the scale transformation is carried out. The phase diagrams of system (6) at different scales of $k=1,3,5$, and 8 with initial values IC $=(1$, $1,1,3), a=1.5, b=0.7$, and $c=0.5$ are shown in Figure 8. The signals $x$ and $z$ are the bipolar signals, so their amplitude changes in both positive and negative directions, but their average value is zero. The output signal $y$ is a unipolar signal, and its amplitude changes positively, and the mean value increases with the change of parameter $k$. The output signal $w$ is a bipolar signal, but it is not regulated. Its mean value is zero, and its amplitude is almost unchanged. The waveform shows the change in the amplitude of signals caused by the correction of parameter $k$ and the effect on the oscillation frequency of the system. The waveforms and average values of $x(t), y(t), z(t)$, and $w(t)$ with parameter $k$ are shown in Figure 9 .

$$
\left\{\begin{array}{l}
\dot{x}=a z|z|-y z, \\
\dot{y}=z^{2}-k^{2} b+k(-0.2+|w|)|z|, \\
\dot{z}=k x-c z|z|, \\
\dot{w}=0.5 z-k w .
\end{array}\right.
$$

\section{Bifurcation Analysis}

When the parameters $a=1.5, b=0.7, d=15, e=10$, and $c \in$ $[0,3]$, system (5) has other coexisting attractors, as shown in Figure 10, where the red trajectory corresponds to the initial condition $\left(\begin{array}{llll}1 & 1 & 0 & 3\end{array}\right)$ and the blue trajectory corresponds to the initial condition $(-110-3)$. It can be seen that the system is periodic when $c=2.5$ and chaos when $c=1.5$. The negative and positive bifurcations show the same Lyapunov exponent except in the region $c=(2.42,2.55)$. The system is chaotic 


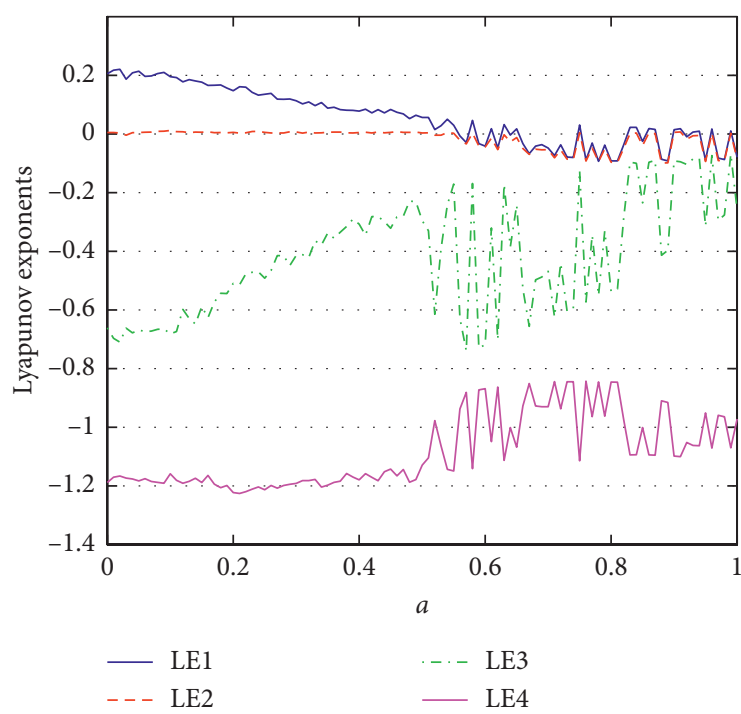

(a)

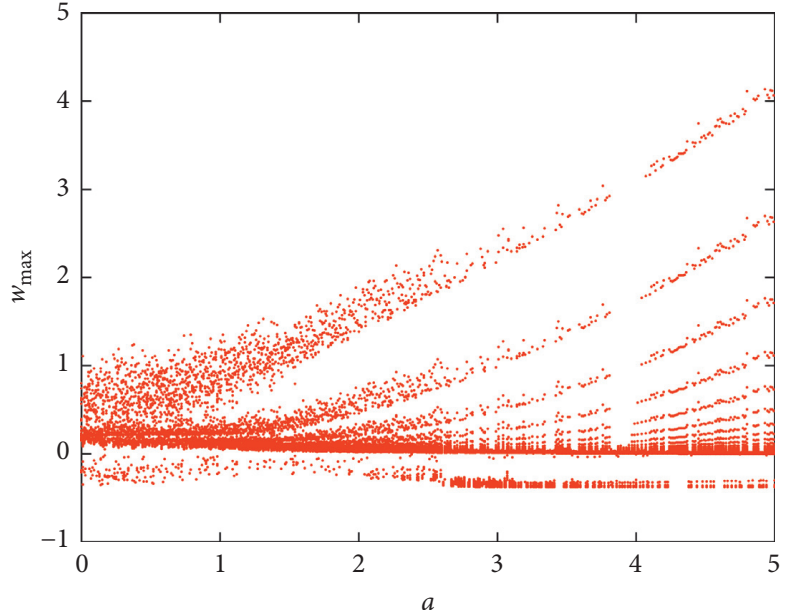

(b)

FIGURE 2: Lyapunov exponent spectrum and bifurcation diagram of the system (2) under different values of the parameter $a$. (a) Lyapunov exponent. (b) Bifurcation diagram.

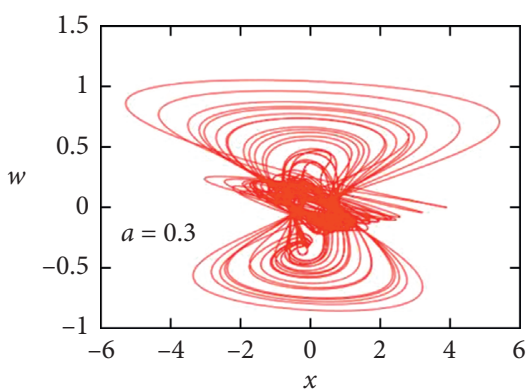

(a)

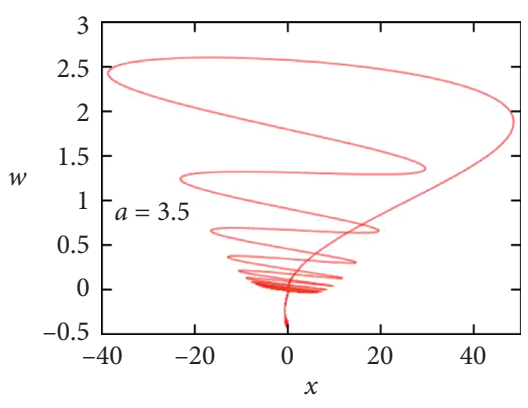

(d)

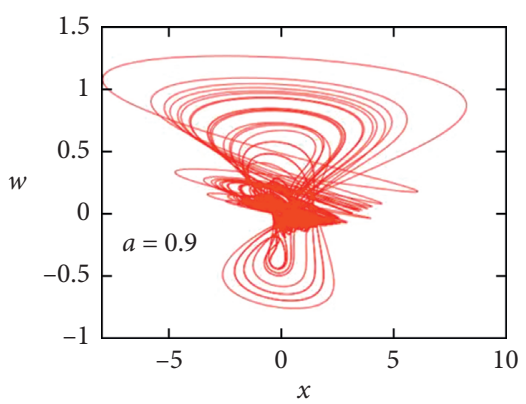

(b)

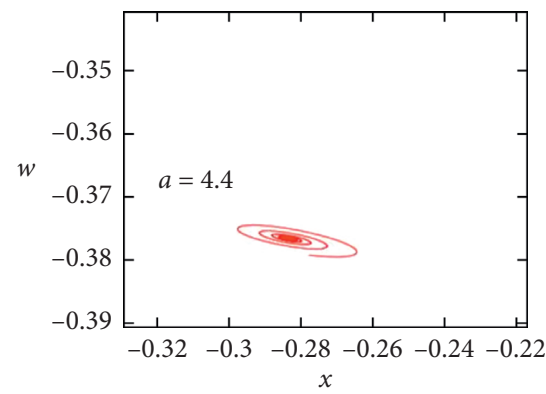

(e)



(c)

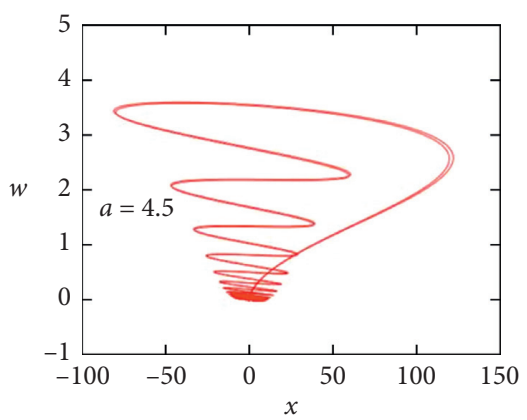

(f)

FIGURe 3: Projections of the system on the $x-w$ phase plane under different values of the parameter $a$. (a) $a=0.3$. (b) $a=0.9$. (c) $a=1.5$. (d) $a=3.5$. (e) $a=4.4$. (f) $a=4.5$.

when $c \in[0.23,2.42]$, and the largest Lyapunov exponent spectrum is positive at the initial values $(1,1,0,3)$. The coexistence period window of different structures appears in a small range near $c=2.5$. The system drops into a periodic window, while $c \in(2.42,2.55)$ at the initial values $(-1,1,0$, $-3)$. The bifurcation diagram of the state variable $x$ and the corresponding Lyapunov exponent are shown in Figure 10.

\section{Circuit Implementation}

In order to verify the chaotic behavior of the system, according to equation (6), the circuit equation is designed as equation (7) and the designed analog circuit diagram is shown in Figure 11. The circuit is composed of four channels, realize the integration, addition, and subtraction 


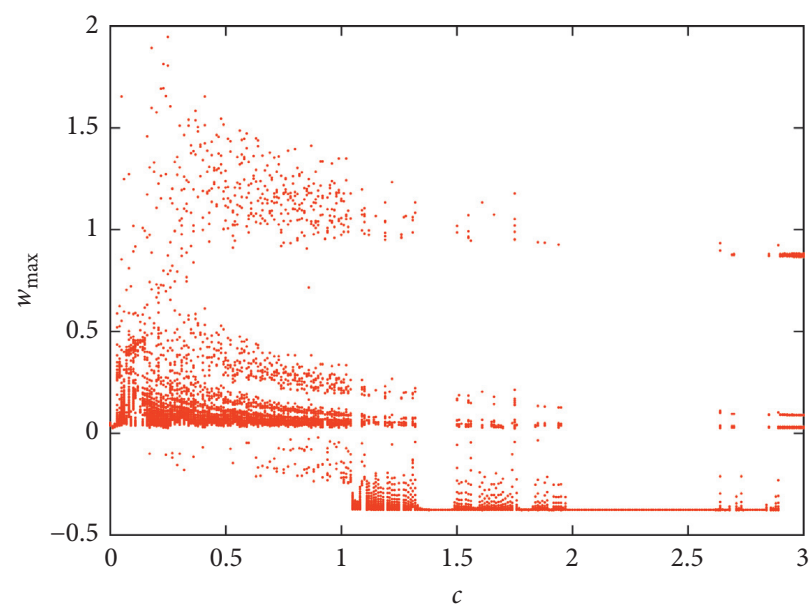

(a)

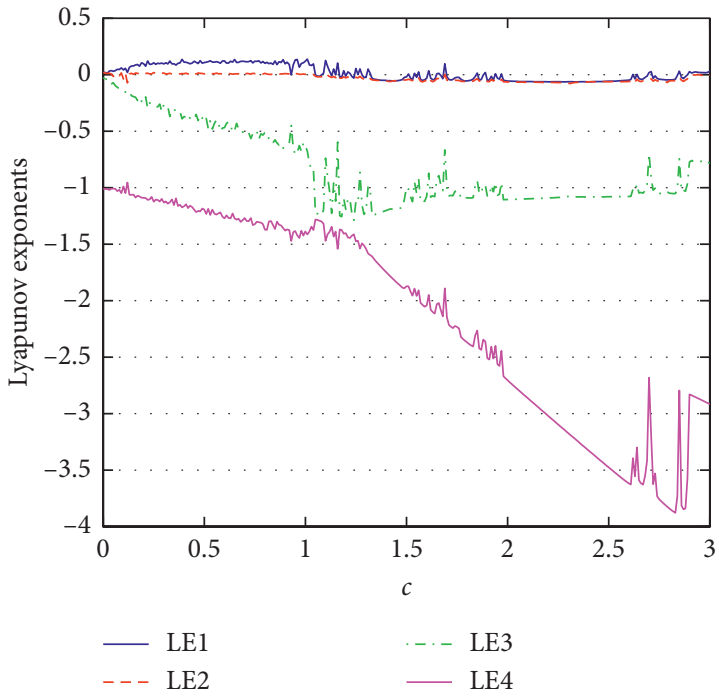

(b)

FIGURE 4: Lyapunov exponent spectrum and bifurcation diagram of system (2) under different values of the parameter $c$. (a) Bifurcation diagram. (b) Lyapunov exponent.

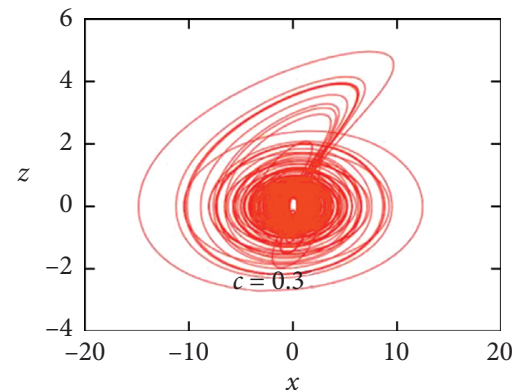

(a)

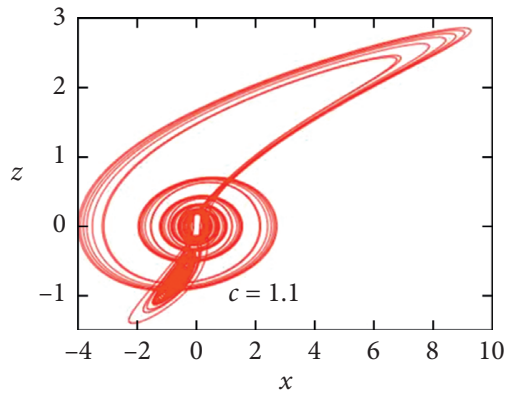

(b)

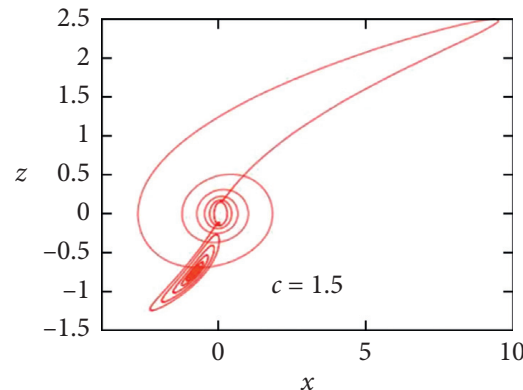

(c)

Figure 5: Projections of the system on the $x-w$ phase plane under different values of the parameter $a$. (a) $c=0.3$. (b) $c=1.1$. (c) $c=1.5$.

of state variables $x, y, z$, and $w$. The operational amplifier 741 performs the addition and integration, and the analog multiplier AD633 performs the nonlinear product operation.

$$
\left\{\begin{array}{l}
C_{x} \frac{\mathrm{d} v_{x}}{\mathrm{~d} t}=\frac{v_{z}\left|v_{z}\right|}{R_{1}}-\frac{v_{y} v_{z}}{R_{8}}, \\
C_{x} \frac{\mathrm{d} v_{y}}{\mathrm{~d} t}=\frac{v_{z} v_{z}}{R_{6}}-\frac{V_{1}}{R_{9}}+\left(-\frac{V_{2}}{R_{27}}+\frac{\left|v_{w}\right|}{R_{27}}\right)\left|v_{z}\right|, \\
C_{z} \frac{\mathrm{d} v_{z}}{\mathrm{~d} t}=\frac{v_{x}}{R_{10}}-\frac{v_{z}\left|v_{z}\right|}{R_{7}}, \\
C_{w} \frac{\mathrm{d} v_{w}}{\mathrm{~d} t}=\frac{v_{z}}{R_{20}}-\frac{v_{w}}{R_{21}},
\end{array}\right.
$$

where $v_{x}, v_{y}, v_{z}, v_{w}$, respectively, indicate the voltage of $x, y$, $z$, and $w$. Let $t=\tau R C$, where $\tau$ is the dimensionless time. The amplitude modulation method in system (6) is used for the scale transformation of the circuit. The parameters are taken as follows: $C_{1}=C_{2}=C_{3}=C_{4}=1 \mathrm{nF}, \quad R_{2}=R_{3}=R_{4}=R_{5}$ $=R_{6}=R_{8}=R_{28}=R_{32}=R=100 \mathrm{k} \Omega, \quad R_{1}=R / a, \quad R_{1}=R / a$, $R_{9}=R /\left(k^{2 *} b\right), \quad R_{27}=R / k, \quad R_{7}=R / c, \quad R_{10}=R / k, \quad R_{20}=2 R$, $R_{21}=R / k, R_{11}=R_{12}=R_{18}=R_{19}=R_{22}=10 \mathrm{k} \Omega$.

The corresponding phase trajectories of system (6) when $k$ is equal to $1 / 3,1$, and 2 , predicted by Multisim simulation are shown in Figure 12. By comparing Figures 12 and 8, it can be seen that the phase diagram of Multisim and MATLAB is basically the same. The difference between the amplitude of the corresponding circuit simulation and the MATLAB numerical simulation is mainly due to the saturation distortion caused by the voltage range of the simulator, which can be solved by scaling the whole system. In this study, in order to verify the appearance of amplitude modulation, amplitude control is realized only by changing the parameter $k$. The results and discussion may be presented separately or in one combined section and may optionally be divided into headed subsections. 


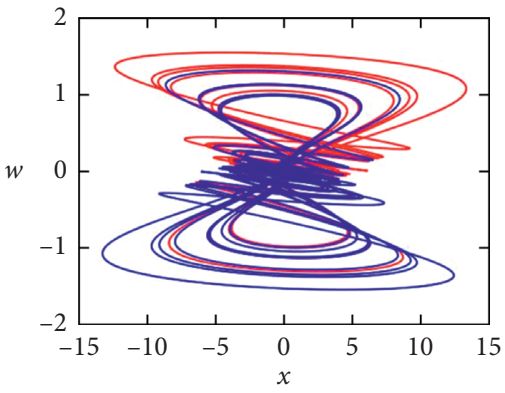

(a)

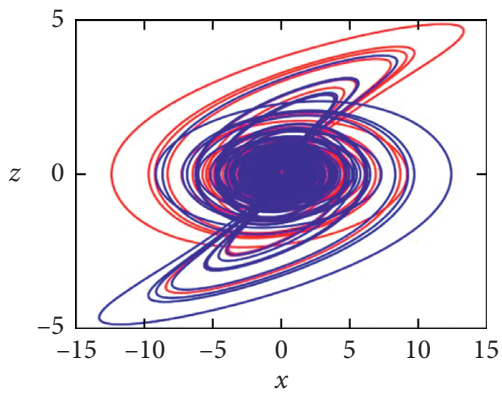

(d)

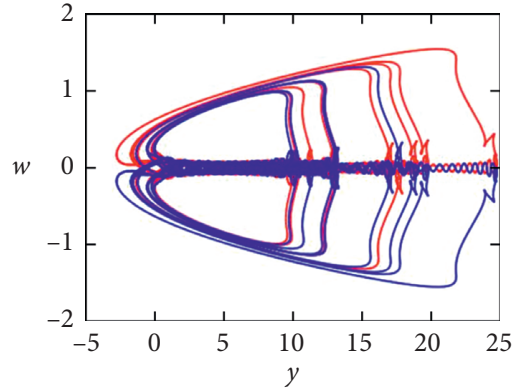

(b)

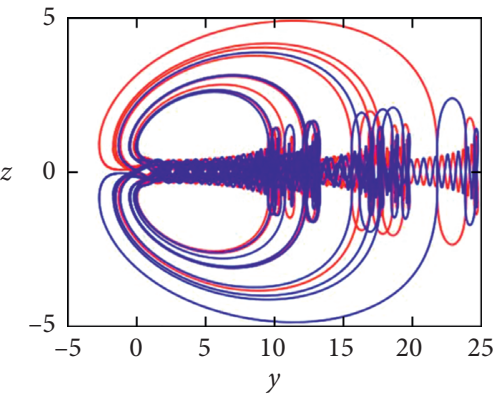

(e)

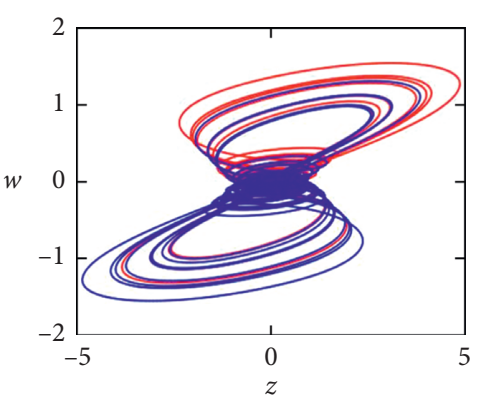

(c)

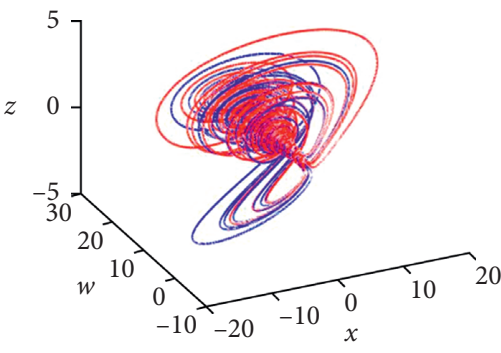

(f)

FIgURE 6: Two attractors with inverse symmetry when IC $=\left(\begin{array}{llll}1 & 1 & 0 & 3\end{array}\right)$ and IC $=\left(\begin{array}{llll}-1 & 1 & 0 & -3\end{array}\right)$. (a) $x-w$. (b) $y-w$. (c) $z-w$. (d) $x-z$. (e) $y-z$. (f) $x-w-z$.

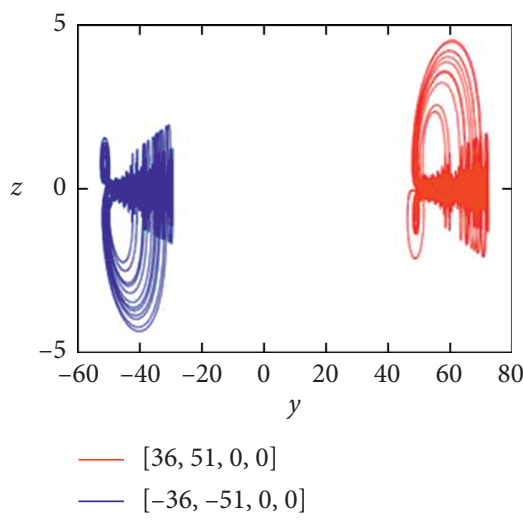

(a)

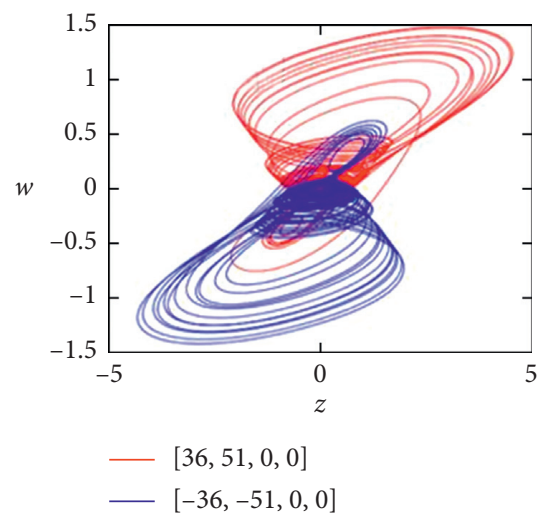

(b)

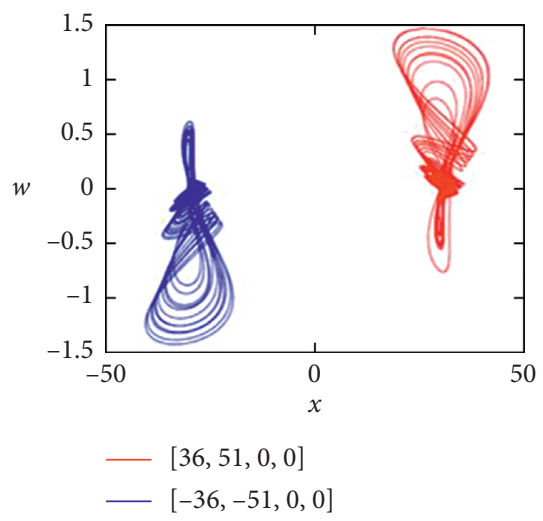

(c)

Figure 7: Conditional symmetric coexisting attractors of system (5). (a) $y-z$. (b) $w-z$. (c) $w-x$.

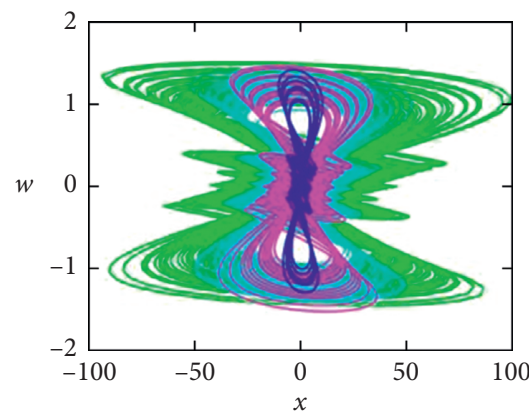

(a)

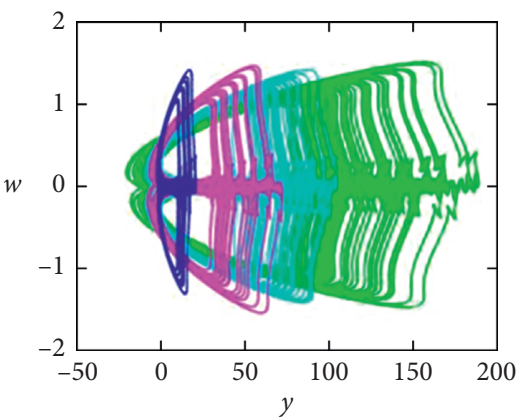

(b)

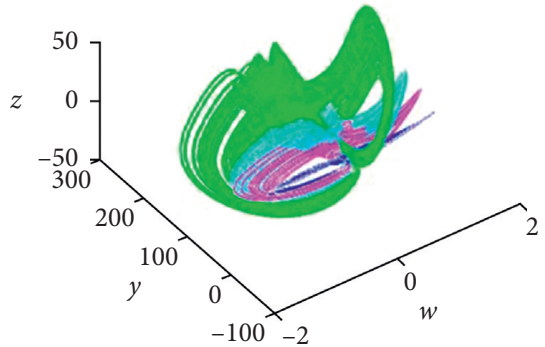

(c)

Figure 8: The phase trajectories of system (7) when $k=1,3,5,8$ are blue, pink, cyan, and green. (a) $x-w$. (b) $y-w$. (c) $y-z-w$. 


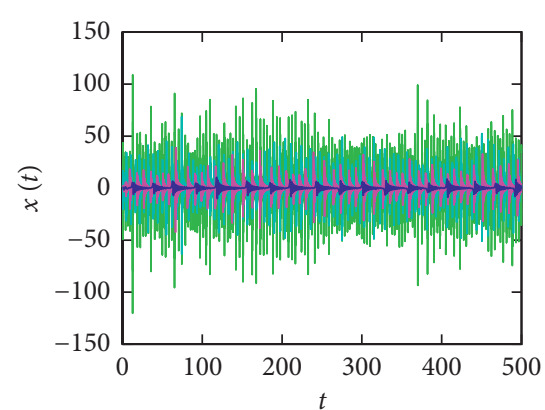

(a)

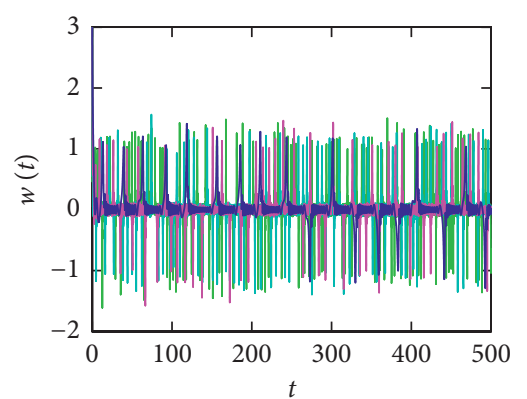

(d)

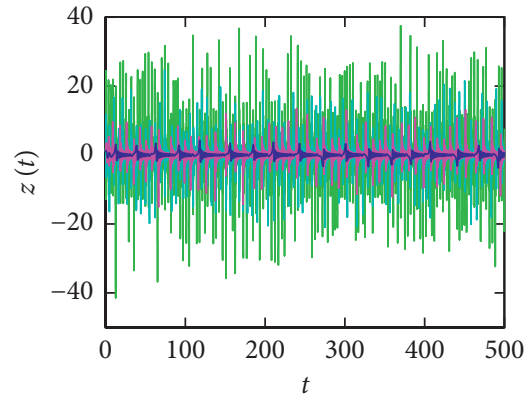

(b)

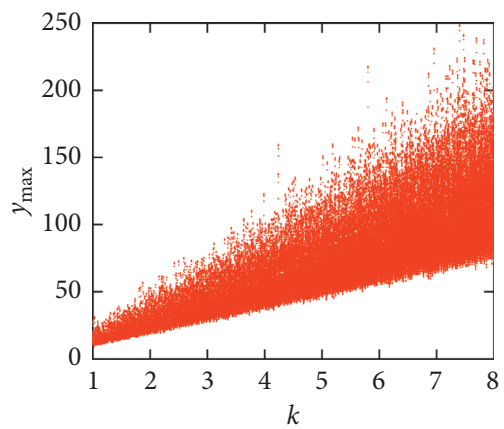

(e)

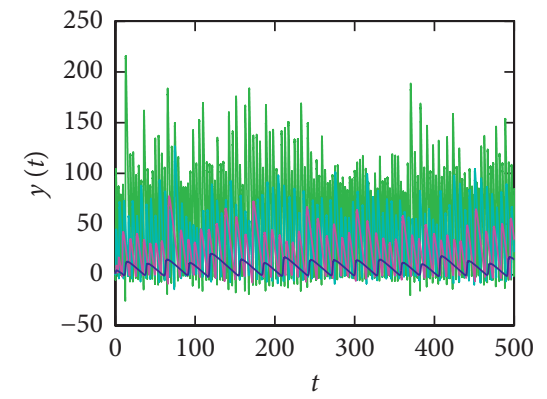

(c)

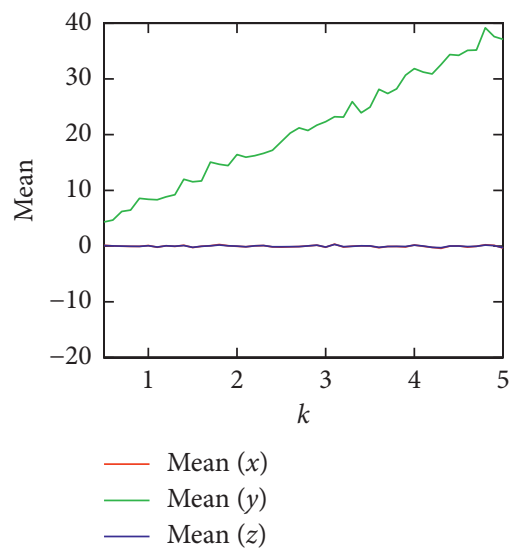

(f)

FIGURE 9: The waveforms and average values of $x(t), y(t), z(t)$, and $w(t)$ with parameter $k$. (a)-(d) Waveforms of $x, y, z$, and $w$. (e) Bifurcation diagram of $k$. (f) Average of $x, y, z$, and $w$.

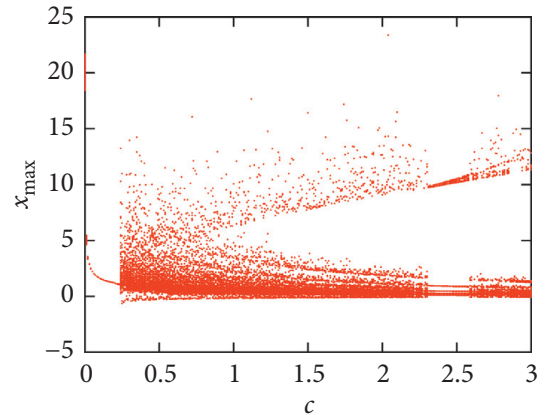

(a)

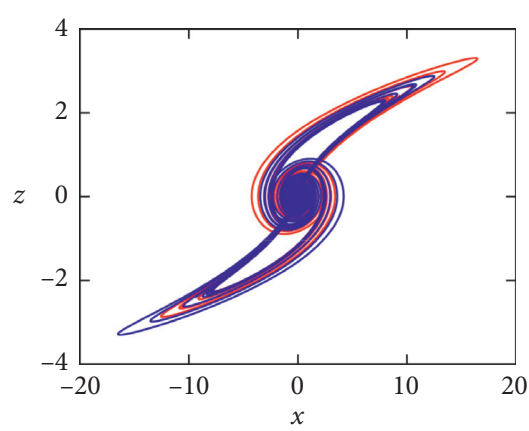

(d)

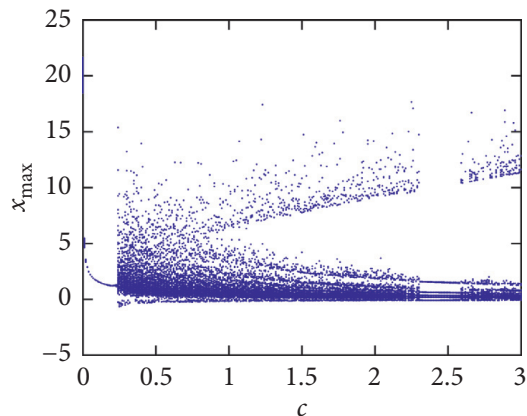

(b)

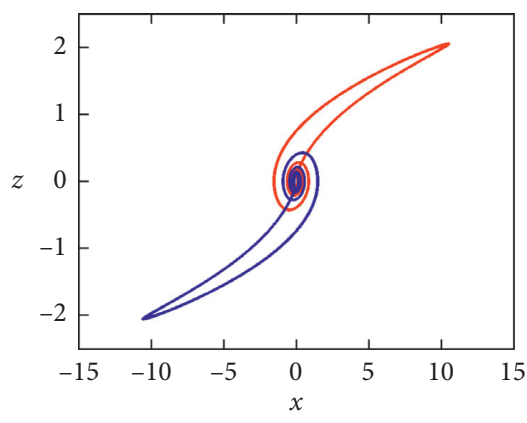

(e)

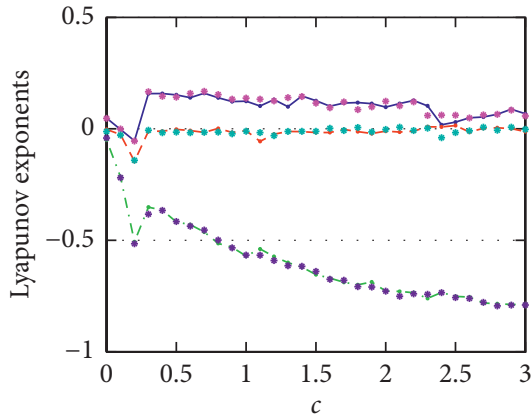

(c)

Figure 10: Bifurcation diagram and Lyapunov exponent spectrums of system (7). (a) IC = $(1,1,0,3)$. (b) IC = (-1, 1, 0, -3$)$. (c) Lyapunov exponent spectrums. (d) $c=1.5$. (e) $c=2.5$. 


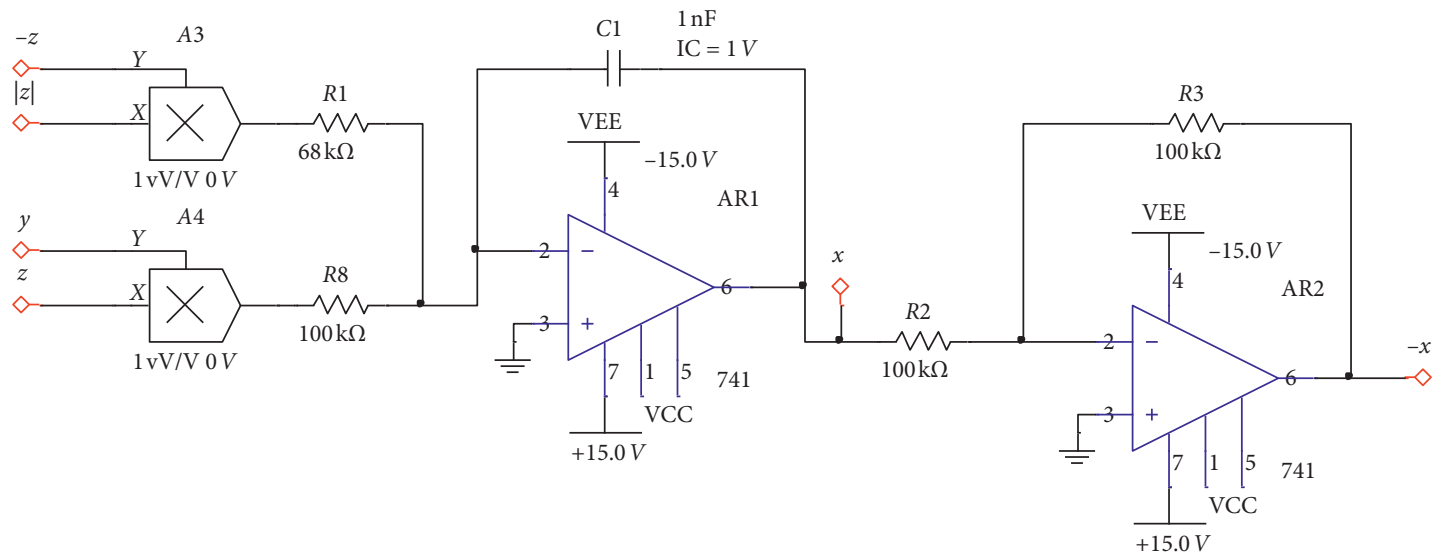

(a)

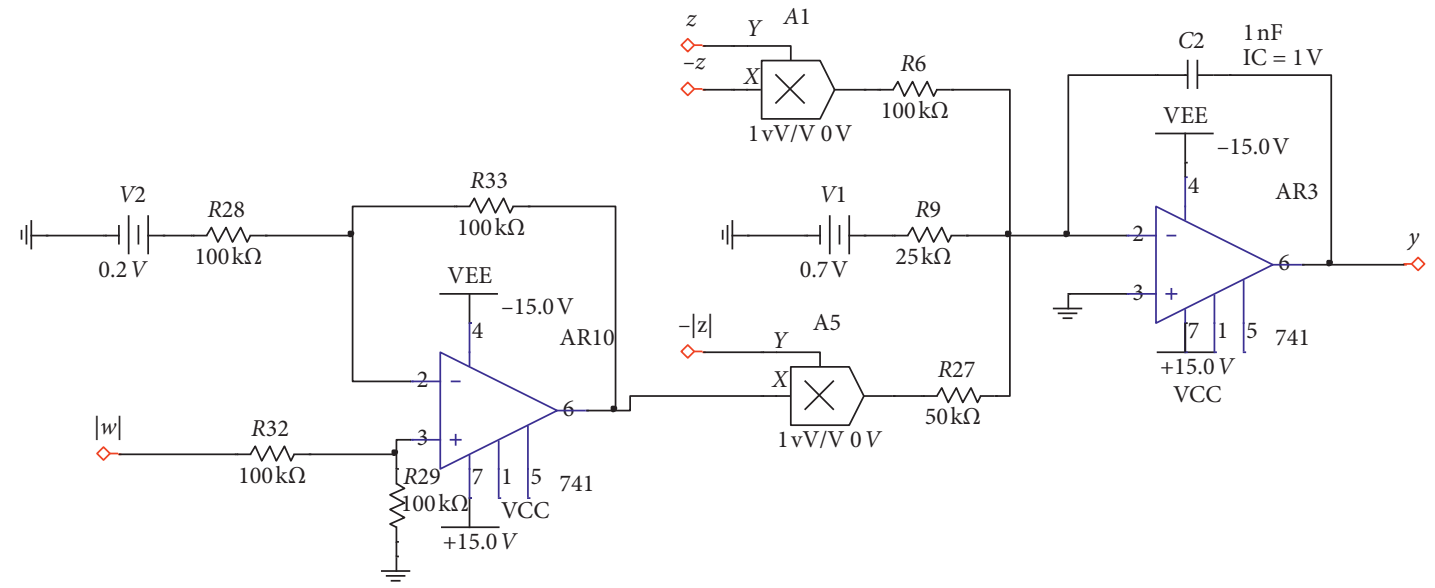

(b)

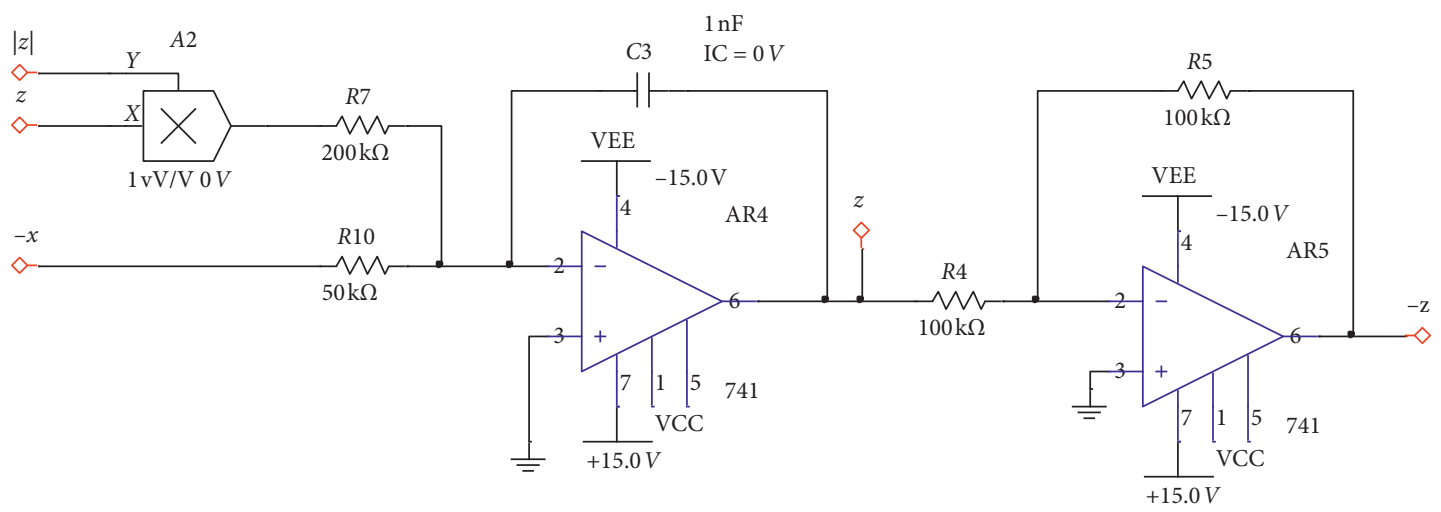

(c)

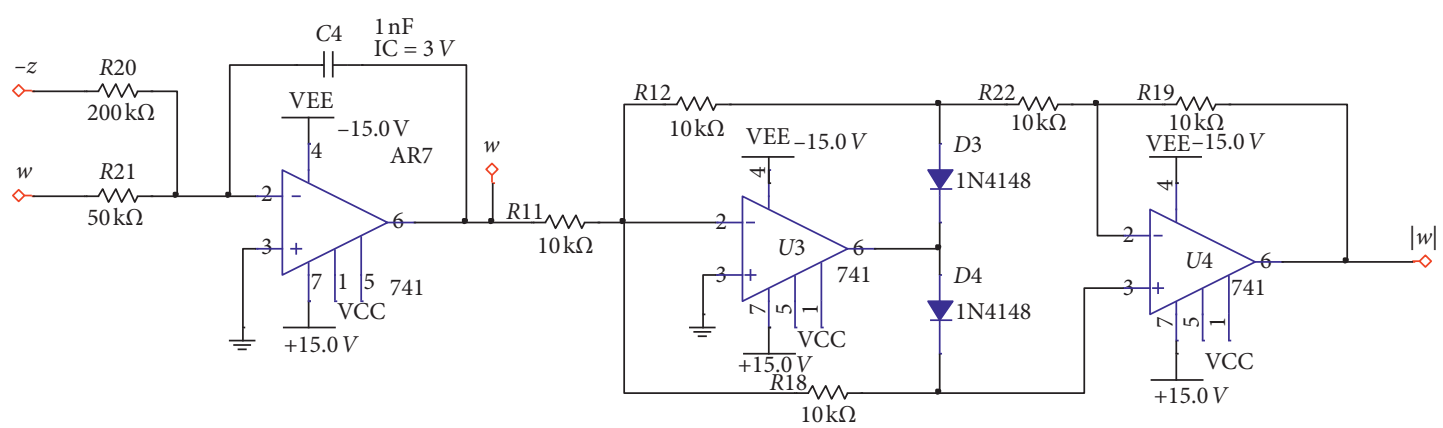

(d)

Figure 11: The circuit schematic of system (6). 

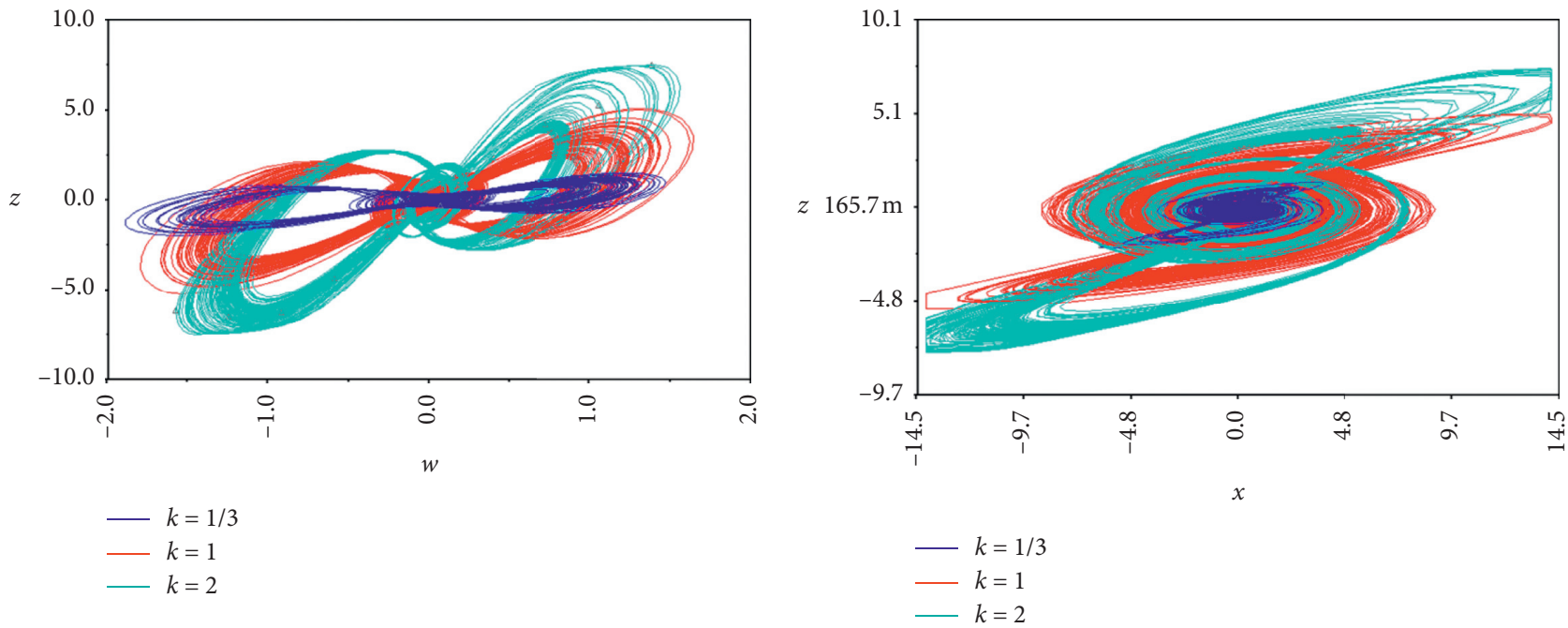

(a)

(b)

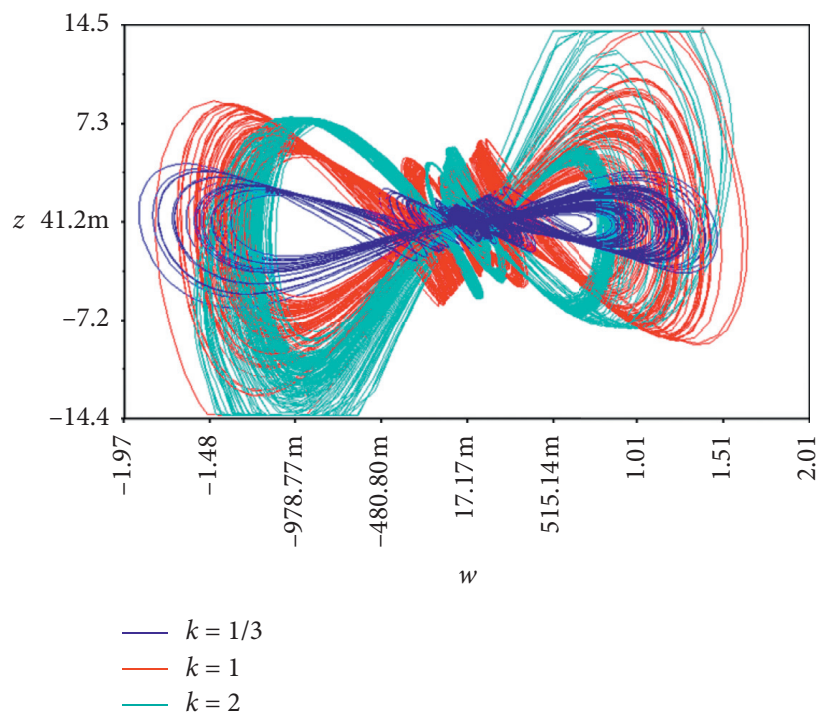

(c)

Figure 12: Phase trajectories of system (6) shown in an oscilloscope while $\mathrm{IC}=(1,1,0,3)$ and $k=1 / 3$ (blue), 1 (red), 2 (cyan).

\section{Conclusions}

In this study, an offset boosting chaotic system was constructed. The dynamical behavior of the system is demonstrated by phase trajectories, Lyapunov exponents, and bifurcation diagrams. The following conclusions are obtained. (1) Absolute value function is introduced in the chaotic system with variable boosting. A chaotic system of conditional symmetry is constructed and analyzed. It is proved that a symmetric system or a conditionally symmetric system can be constructed by using offset boosting. (2) The dynamic characteristics of the new system generated by scale change are the same as that of the original system, but a nonbifurcation parameter can be formed to adjust the amplitude and frequency of system (3). The simulation circuit verifies the amplitude modulation characteristics of the system.

\section{Data Availability}

The data used to support the findings of this study are available from the corresponding author upon request.

\section{Conflicts of Interest}

The authors declare that there are no conflicts of interest.

\section{Acknowledgments}

This work was supported by Shandong Province Key Research and Development Plan (2019GGX104092) and the Natural Science Foundation of Shandong Province (ZR2020KA007). 


\section{References}

[1] D. B. Strukov, G. S. Snider, D. R. Stewart, and R. S. Williams, "The missing memristor found," Nature, vol. 453, no. 7191, pp. 80-83, 2008.

[2] M. E. Sahin, Z. G. Cam Taskiran, H. Guler, and S. E. Hamamci, "Simulation and implementation of memristive chaotic system and its application for communication systems," Sensors and Actuators A: Physical, vol. 290, pp. 107-118, 2019.

[3] Z. Rahman, H. Al-Kashoash, S. Ramadhan et al., "Adaptive control synchronization of a novel memristive chaotic system for secure communication applications," Inventions, vol. 4, no. 2, p. 30, 2019.

[4] X. Shi, S. Duan, L. Wang, T. Huang, and C. Li, "A novel memristive electronic synapse-based hermite chaotic neural network with application in cryptography," Neurocomputing, vol. 166, pp. 487-495, 2015.

[5] C. S. Kuka, Y. Hu, Q. Xu, and M. Alkahtani, "An innovative near-field communication security based on the chaos generated by memristive circuits adopted as symmetrical key," IEEE Access, vol. 8, pp. 167975-167984, 2020.

[6] J. Sun, C. Li, T. Lu et al., "A memristive chaotic system with hypermultistability and its application in image encryption," IEEE Access, vol. 8, pp. 139289-139298, 2020.

[7] Y. Yang, L. Wang, S. Duan et al., "Dynamical analysis and image encryption application of a novel memristive hyperchaotic system," Optics \& Laser Technology, vol. 133, p. 106553, 2021.

[8] J. Yang, L. Wang, Y. Wang et al., "A novel memristive hopfield neural network with application in associative memory," Neurocomputing, vol. 227, pp. 142-148, 2016.

[9] L. Wang and H. Zou, "A new emotion model of associative memory neural network based on memristor," Neurocomputing, vol. 410, pp. 83-92, 2020.

[10] S. Vaidyanathan, A. T. Azar, A. Akgul, C. H. Lien, S. Kacar, and U. Cavusoglu, "A memristor-based system with hidden hyperchaotic attractors, its circuit design, synchronisation via integral sliding mode control and an application to voice encryption," International Journal of Automation and Control, vol. 13, no. 6, pp. 644-667, 2019.

[11] J. Sun, X. Xiao, Q. Yang et al., "Memristor-based hopfield network circuit for recognition and sequencing application," AEU-International Journal of Electronics and Communications, p. 153698, 2021.

[12] N. Wang, G. Zhang, and H. Bao, "Bursting oscillations and coexisting attractors in a simple memristor-capacitor-based chaotic circuit," Nonlinear Dynamics, vol. 97, no. 2, pp. 1477-1494, 2019.

[13] N. Wang, C. Li, H. Bao, M. Chen, and B. Bao, "Generating multi-scroll Chua's attractors via simplified piecewise-linear Chua's diode," IEEE Transactions on Circuits and Systems I: Regular Papers, vol. 66, no. 12, pp. 4767-4779, 2019.

[14] B. Lai and J. He, "Dynamic analysis, circuit implementation and passive control of a novel four-dimensional chaotic system with multiscroll attractor and multiple coexisting attractors," Pramana, vol. 90, p. 33, 2018.

[15] S. Jafari, V. T. Pham, and T. Kapitaniak, "Multiscroll chaotic sea obtained from a simple 3D system without equilibrium," International Journal of Bifurcation and Chaos: In Applied Sciences and Engineering, vol. 26, no. 2, p. 1650031, 2016.

[16] S. Jafari, J. C. Sprott, V.-T. Pham, C. Volos, and C. Li, "Simple chaotic 3D flows with surfaces of equilibria," Nonlinear Dynamics, vol. 86, no. 2, pp. 1349-1358, 2016.
[17] S. Zhang, X. Wang, and Z. Zeng, "A simple no-equilibrium chaotic system with only one signum function for generating multidirectional variable hidden attractors and its hardware implementation," Chaos, vol. 30, no. 5, p. 053129, 2020.

[18] Q. Lai, "A unified chaotic system with various coexisting attractors," International Journal of Bifurcation and Chaos: In Applied Sciences and Engineering, vol. 31, no. 1, p. 2150013, 2021.

[19] R. L. M. Tagne, J. Kengne, and A. N. Negou, "Multistability and chaotic dynamics of a simple jerk system with a smoothly tuneable symmetry and nonlinearity," International Journal of Dynamics and Control, vol. 7, no. 2, pp. 476-495, 2019.

[20] C. Ma, J. Mou, L. Xiong, S. Banerjee, T. Liu, and X. Han, "Dynamical analysis of a new chaotic system: asymmetric multistability, offset boosting control and circuit realization," Nonlinear Dynamics, vol. 103, no. 3, pp. 2867-2880, 2021.

[21] H. Bao, W. Liu, and M. Chen, "Hidden extreme multistability and dimensionality reduction analysis for an improved nonautonomous memristive FitzHugh-Nagumo circuit," Nonlinear Dynamics, vol. 96, no. 3, pp. 1879-1894, 2019.

[22] G. D. Leutcho and J. Kengne, "A unique chaotic snap system with a smoothly adjustable symmetry and nonlinearity: chaos, offset-boosting, antimonotonicity, and coexisting multiple attractors," Chaos, Solitons \& Fractals, vol. 113, pp. 275-293, 2018.

[23] C. Li, J. C. Sprott, and H. Xing, "Constructing chaotic systems with conditional symmetry," Nonlinear Dynamics, vol. 87, no. 2, pp. 1351-1358, 2017.

[24] C. Li, J. C. Sprott, Z. Yuan et al., "Constructing chaotic systems with total amplitude control," International Journal of Bifurcation and Chaos, vol. 25, no. 10, p. 1530025, 2015.

[25] C. Li, J. C. Sprott, and H. Xing, "Crisis in amplitude control hides in multi-stability," International Journal of Bifurcation and Chaos, vol. 26, no. 14, p. 1650233, 2016.

[26] A. Bayani, K. Rajagopal, A. J. M. Khalaf et al., "Dynamical analysis of a new multistable chaotic system with hidden attractor: antimonotonicity, coexisting multiple attractors, and offset boosting," Physics Letters A, vol. 383, pp. 14501456, 2019.

[27] H. Zang, L. Huang, Y. Wang et al., "Study on bias control of memristor multistablity system," E3S Web of Conferences, vol. 233, p. 04002, 2021.

[28] L. Huang, T. Lei, H. Bian et al., "Amplitude control and polarity switching in a memristive chaotic oscillator," in Proceedings of the 2020 Chinese Automation Congress (CAC), Shanghai, China, November 2020.

[29] C. Li, T. Lei, X. Wang, and G. Chen, "Dynamics editing based on offset boosting," Chaos, vol. 30, no. 6, p. 063124, 2020.

[30] N. Wang, G. Zhang, and H. Li, "Parametric control for multiscroll attractor generation via nested sine-PWL function," IEEE Transactions on Circuits and Systems II: Express Briefs, vol. 68, no. 3, pp. 1033-1037, 2021.

[31] N. Wang, G. Zhang, N. V. Kuznetsov et al., "Hidden attractors and multistability in a modified Chua's circuit," Communications in Nonlinear Science and Numerical Simulation, vol. 92, p. 105494, 2021.

[32] X. Zhang, C. Li, Y. Chen et al., "A memristive chaotic oscillator with controllable amplitude and frequency," Chaos, Solitons \& Fractals, vol. 139, p. 110000, 2020.

[33] Z. Gu, C. Li, H. H. C. Iu et al., "Constructing hyperchaotic attractors of conditional symmetry," The European Physical Journal B, vol. 92, no. 10, p. 221, 2019.

[34] C. Li, J. C. Sprott, Y. Liu et al., "Offset boosting for breeding conditional symmetry," International Journal of Bifurcation 
and Chaos: In Applied Sciences and Engineering, vol. 28, no. 14, p. $1850163,2018$.

[35] B. C. Bao, Q. Xu, H. Bao, and M. Chen, "Extreme multistability in a memristive circuit," Electronics Letters, vol. 52, no. 12, pp. 1008-1010, 2016.

[36] F. Yuan, G. Wang, and X. Wang, "Extreme multistability in a memristor-based multi-scroll hyper-chaotic system," Chaos, vol. 26, no. 7, p. 073107, 2016.

[37] B. C. Bao, H. Bao, N. Wang, M. Chen, and Q. Xu, "Hidden extreme multistability in memristive hyperchaotic system," Chaos, Solitons \& Fractals, vol. 94, pp. 102-111, 2017.

[38] C. Li and J. C. Sprott, "Amplitude control approach for chaotic signals," Nonlinear Dynamics, vol. 73, no. 3, pp. 1335-1341, 2013.

[39] J. Gu, C. Li, Y. Chen, H. H. C. Iu, and T. Lei, "A conditional symmetric memristive system with infinitely many chaotic attractors," IEEE Access, vol. 8, pp. 12394-12401, 2020.

[40] C. Li, W. Joo-Chen Thio, H. Ho-Ching Iu, and T. Lu, "A memristive chaotic oscillator with increasing amplitude and frequency,” IEEE Access, vol. 6, pp. 12945-12950, 2018.

[41] H. Zang, Z. Gu, T. Lei et al., "Coexisting chaotic attractors in a memristive system and their amplitude control," Pramana, vol. 94 , no. 4 , p. $62,2020$.

[42] C. Li and J. C. Sprott, "Variable-boostable chaotic flows," Optik, vol. 127, no. 22, pp. 10389-10398, 2016. 